\title{
Successful treatment of refractory listeria meningitis and bacteremia with trimethoprim-sulfamethoxazole in an immunocompetent child
}

\author{
Meltem Polat ${ }^{1}$, Soner Sertan Kara ${ }^{1}$, Anıl Tapısız¹, Okşan Derinöz ${ }^{2}$, Kayhan Çağlar ${ }^{3}$ \\ Hasan Tezer ${ }^{1}$ \\ Divisions of ${ }^{1}$ Pediatric Infectious Diseases, and ${ }^{2}$ Pediatric Emergency, Department of Pediatrics and ${ }^{3}$ Department of \\ Microbiology, Gazi University Faculty of Medicine, Ankara, Turkey. \\ E-mail: meltemtemizhan@gmail.com \\ Received: 5 June 2015, Revised: 29 July 2015, Accepted: 29 September 2015
}

SUMMARY: Polat M, Kara SS, Tapısız A, Derinöz O, Çağlar K, Tezer H. Successful treatment of refractory listeria meningitis and bacteremia with trimethoprim-sulfamethoxazole in an immunocompetent child. Turk J Pediatr 2016; 58: 220-222.

Listeria monocytogenes is an important cause of life-threatening bacteremia and meningoencephalitis in neonates, pregnant women, the elderly, and immunocompromised individuals. However, it is an uncommon cause of illness in immunocompetent children beyond the neonatal period. Ampicillin with or without an aminoglycoside remains the best treatment for listeriosis. Here, we report a rare case of Listeria meningitis and bacteremia in a 7-month-old immunocompetent girl, which was refractory to ampicillin plus gentamicin treatment and successfully treated by the addition of TMP/SMX.

Key words: immunocompetent child, Listeria meningitis, trimethoprim-sulfamethoxazole.

The bacterium, Listeria monocytogenes ( $L$. monocytogenes), is an important food-borne cause of life-threatening bacteremia and central nervous system (CNS) infection in certain high risk groups such as neonates, elderly people, pregnant women, and immunosuppressed patients. However, it is an uncommon cause of illness in immunocompetent children beyond the neonatal period ${ }^{1-3}$.

L. monocytogenes is difficult to isolate and is not susceptible to third-generation cephalosporins commonly used for the empirical treatment of bacterial meningitis. Ampicillin alone or in combination with an aminoglycoside is the first line treatment for L. monocytogenes infections ${ }^{1,4}$. However, treatment failures were previously reported with ampicillin plus aminoglycoside regimen. ${ }^{5}$ In patients who fail to respond to therapy, there is now increasing evidence to conclude that trimethoprim-sulfamethoxazole (TMP/SMX) may be the best alternative option $^{5-7}$. We herein report a rare case of $L$. monocytogenes meningitis and bacteremia in a 7-month-old immunocompetent child, which was unresponsive to ampicillin plus gentamicin treatment and improved dramatically after the substitution of gentamicin by TMP/SMX.

\section{Case Report}

A previously healthy, 7-month-old girl was admitted to our hospital due to persistent fever up to $40^{\circ} \mathrm{C}$, vomiting and diarrhea lasting for 3 days followed by strabismus that was noticed by her mother 1 day prior to admission. There was a history of eating an undercooked meatballs 2 days before the onset of symptoms. The physical examination revealed fontanel bulging and bilateral abducens nerve palsy (Fig. 1a). Initial laboratory investigations showed elevated white blood cell (WBC) count of $19.3 \times 10^{9}$ cells/L ( $80 \%$ neutrophils, $15 \%$ monocytes, $5 \%$ lymphocytes), and C-reactive protein (CRP) level of $52 \mathrm{mg} / \mathrm{dl}$. Cerebrospinal fluid (CSF) analysis revealed 1350 cells $/ \mathrm{mm}^{3}$ (80\% lymphocytes, $20 \%$ neutrophils), with a glucose and protein concentration of 42 and 132 $\mathrm{mg} / \mathrm{dl}$, respectively. Both computed tomography scan and magnetic resonance imaging of the brain were normal. Empiric treatment with 
intravenous (IV) ceftriaxone $(100 \mathrm{mg} / \mathrm{kg}$ per day) and vancomycin (60 mg/kg per day) was initiated with the aim of targeting the most common pathogens of bacterial meningitis. However, her fever persisted. On the $4^{\text {th }}$ day of hospitalization, admission blood and CSF cultures grew diphtheroids subsequently identified as L. monocytogenes, fully susceptible in vitro to penicillin, ampicillin, gentamicin, TMP/SMX, and meropenem. Treatment was changed to IV ampicillin $(300 \mathrm{mg} / \mathrm{kg}$ per day) plus gentamicin $(7.5 \mathrm{mg} / \mathrm{kg}$ per day). On hospital day 9 , after 5 days of ampicillin plus gentamicin treatment, the patient was still highly febrile with strabismus, fontanel bulging, vomiting, and markedly elevated CRP values. Follow-up blood cultures and a repeat CSF culture remained positive for L. monocytogenes. Echocardiographic examination for infective endocarditis was found to be normal. Because of the apparent lack of clinical and microbiological response to treatment, high-dose IV TMP/SMX (20 mg/kg per day of TMP) was substituted for gentamicin. Her clinical condition improved markedly within 2 days, and control blood and CSF cultures became negative 5 days after the initiation of TMP-SMX. Overall, the patient was treated with ampicillin for 26 days and TMP/SMX for 21 days. The patient was discharged in good condition on day 30 . Her strabismus resolved completely two weeks after discharge (Fig. 1b). During follow-up, all immunological investigations were found to be normal, including peripheral blood lymphocyte subsets, in vitro lymphoproliferative response to mitogens, flow cytometric analysis of interleukin (IL)-12R $\beta 1$ cell surface expression on activated $\mathrm{T}$ cells, and IFN- $\gamma$ cell surface expression on monocytes. Serological testing for HIV was non-reactive.

\section{Discussion}

Listeria monocytogenes is a facultative, intracellular, gram-positive bacillus that is the agent of listeriosis, a serious infection usually caused by the ingestion of contaminated food. Meningitis and septicemia are the most commonly observed clinical presentations of listeriosis; however, they are rare in immunocompetent children ${ }^{1-3,8}$. Herein we described a rare case of $L$. monocytogenes meningitis and bacteremia in an immunocompetent child that was probably infected through ingestion of undercooked meat.

In clinical specimens, the organisms can be gram-variable and can resemble diphtheroids, cocci, or diplococci. Laboratory misidentification as diphtheroids, streptococci, or enterococci is not uncommon, and the isolation of a "diphtheroid" from blood or CSF should always alert one to the possibility that the organism may be L. monocytogenes. L. monocytogenes is isolated from the blood in $40-75 \%$ of cases of meningitis due to the organism ${ }^{1}$.

Listeria infections are associated with a high mortality rate, and thus effective antibiotic treatment is essential. Although Listerias are uniformly susceptible in vitro to most common antibiotics except cephalosporins, ampicillin alone or in combination with gentamicin remains the treatment of choice. However, some patients may require alternative therapies due to penicillin intolerance or unresponsiveness to initial treatment. In such cases, TMP/SMX can be used as alternative therapy ${ }^{1,4}$.

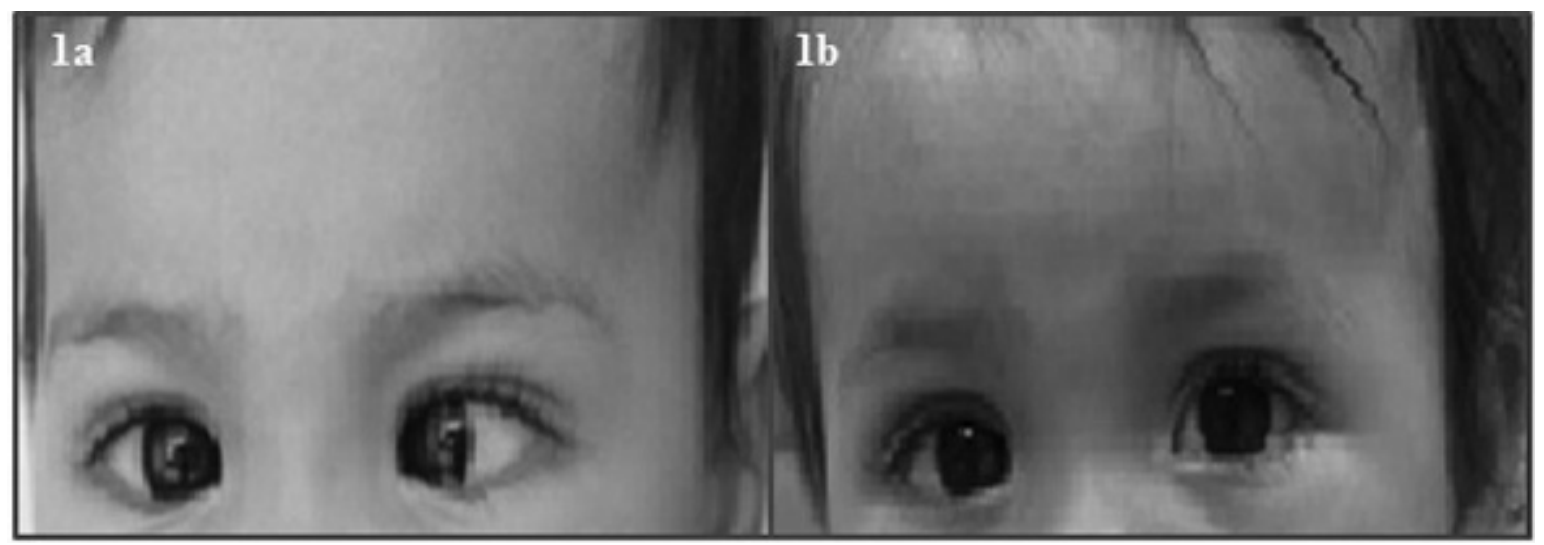

Fig. 1. Bilateral abducens nerve palsy at the time of admission (a), and complete improvement after treatment (b). 
In this case, despite in vitro susceptibility result, we could not eradicate the microorganism in vivo. As in our case, treatment failures were previously reported with ampicillin plus aminoglycoside regimen in Listeria meningoencephalitis ${ }^{5,9}$. Ampicillin and gentamicin, although synergistic and bactericidal against Listeria in vitro, unfortunately have limited intracellular penetration and activity ${ }^{10}$. In contrast, TMP/SMX has bactericidal extracellular and intracellular activity against Listeria and penetrates well into $\mathrm{CNS}^{10}$. In a retrospective study, it was found that the combination of TMP/SMX and ampicillin was associated with a significantly lower failure rate and a lower mortality rate than the combination of ampicillin and aminoglycoside ${ }^{5}$. Additionally, L. monocytogenes is located mainly within macrophages and parenchymal cells of the spleen and liver, hardly accessible to certain drugs, such as ampicillin and gentamicin ${ }^{10}$, which may be a reason for the persistent bacteremia in our patient.

Although L. monocytogenes is a rare cause of meningitis and bacteremia in previously healthy children, it should be considered when treating a child with meningitis that is unresponsive to empiric antibiotic treatment with thirdgeneration cephalosporins and when blood, CSF or other normally sterile specimen reported to have "diphtheroids" on Gram stain or culture. TMP/SMX can be effective for the treatment of CNS listeriosis refractory to conventional treatment.

\section{REFERENCES}

1. Lorber B. Listeria monocytogenes. In: Long SS, Pickering LK, Prober CG (ed). Principles and Practice of Pediatric Infectious Diseases ( $4^{\text {th }}$ ed). Philadelphia: Elsevier Inc; 2012: 762-767.

2. Ben Shimol S, Einhorn M, Greenberg D. Listeria meningitis and ventriculitis in an immunocompetent child: case report and literature review. Infection 2012; 40: 207-211.

3. Eminoğlu FT, Küçükçongar A, Yılmaz FB, Aktaş A, Hasanoğlu A. Dokuz yaşındaki kız hastada listeria menenjiti. Turkiye Klinikleri J Pediatr 2008; 17: 183185.

4. Temple ME, Nahata MC. Treatment of listeriosis. Ann Pharmacother 2000; 34: 656-661.

5. Merle-Melet M, Dossou-Gbete L, Maurer P, et al. Is amoxicillin-cotrimoxazole the most appropriate antibiotic regimen for Listeria meningoencephalitis? Review of 22 cases and the literature. J Infect 1996; 33: $79-85$.

6. Wacker P, Ozsahin H, Groll AH, Reinhard L, Humbert J. Trimethoprim-sulfamethoxazole salvage for refractory listeriosis during maintenance chemotherapy for acute lymphoblastic leukemia. J Pediatr Hematol Oncol 2000; 22: $340-343$

7. Spitzer PG, Hammer SM, Karchmer AW. Treatment of Listeria monocytogenes infection with trimethoprimsulfamethoxazole: case report and review of the literature. Rev Infect Dis 1986; 8: 427.

8. Doganay M. Listeriosis: clinical presentation. FEMS Immunol Med Microbiol 2003; 35: 173-175.

9. McLauchlin J, Audurier A, Taylor AG. Treatment failure and recurrent human listeriosis. J Antimicrob Chemother 1991; 27: 851- 857.

10. Hof H, Nichterlein T, Kretschmar M. Management of listeriosis. Clin Microbiol Rev 1997; 10: 345-357. 